\title{
Research into the Dynamic Development Trend of the Competitiveness of China's Regional Construction Industry
}

\author{
Bingsheng Liu*, Xueqing Wang**, Chunlai Chen***, and Zhijie Ma****
}

\section{Erratum to:KSCE Journal of Civil Engineering (2014) 18(1):1-10 DOI 10.1007/s12205-014-0048-7}

The original version of this article unfortunately contained a mistake.

Errata:

1. Table 5, Trend Analysis of the Competitiveness of China's Regional Construction Industry:

\begin{tabular}{|c|c|c|c|c|c|}
\hline & 2005 & 2006 & 2007 & 2008 & Trend \\
\hline Beijing & $1.3417(5)$ & $1.1548(5)$ & $1.1451(5)$ & $0.7964(5)$ & - \\
\hline Tianjin & $-0.4023(16)$ & $-0.4226(15)$ & $-0.3919(16)$ & $-0.3884(16)$ & - \\
\hline Hebei & $0.1780(10)$ & $0.1241(11)$ & $0.1031(11)$ & $0.0390(11)$ & \\
\hline Shanxi & $-0.3914(15)$ & $-0.4604(16)$ & $-0.4502(17)$ & $-0.4236(18)$ & \\
\hline Inner Mongolia & $-0.7310(23)$ & $-0.6884(22)$ & $-0.6963(22)$ & $-0.7443(24)$ & \\
\hline Liaoning & $0.5201(7)$ & $0.4597(7)$ & $0.4644(7)$ & $0.3884(8)$ & \\
\hline Jilin & $-0.6969(21)$ & $-0.7383(24)$ & $-0.7317(24)$ & $-0.7103(22)$ & \\
\hline Heilongjiang & $-0.7011(22)$ & $-0.6579(20)$ & $-0.6941(21)$ & $-0.6308(19)$ & \\
\hline Shanghai & $0.7601(6)$ & $0.7473(6)$ & $0.5989(6)$ & $0.5979(6)$ & - \\
\hline Jiangsu & $2.6186(1)$ & $2.7535(1)$ & $2.8260(1)$ & $3.0233(1)$ & - \\
\hline Zhejiang & $1.7875(2)$ & $1.8626(2)$ & $1.8101(2)$ & $1.7941(2)$ & - \\
\hline Anhui & $-0.1650(14)$ & $-0.1112(14)$ & $-0.0933(14)$ & $-0.0412(14)$ & - \\
\hline Fujian & $-0.1318(13)$ & $-0.0169(12)$ & $0.0287(12)$ & $0.0314(12)$ & \\
\hline Jiangxi & $-0.6568(20)$ & $-0.6638(21)$ & $-0.6877(20)$ & $-0.6736(20)$ & - \\
\hline Shandong & $1.6190(4)$ & $1.5790(4)$ & $1.4998(4)$ & $1.5355(4)$ & - \\
\hline Henan & $0.1618(11)$ & $0.2956(9)$ & $0.4355(8)$ & $0.5434(7)$ & \\
\hline Hubei & $0.2331(9)$ & $0.2628(10)$ & $0.3351(9)$ & $0.2533(10)$ & \\
\hline Hunan & $-0.0662(12)$ & $-0.0460(13)$ & $-0.0644(13)$ & $-0.0374(13)$ & \\
\hline Guangdong & $1.7787(3)$ & $1.6706(3)$ & $1.6938(3)$ & $1.6539(3)$ & - \\
\hline Guangxi & $-0.7521(24)$ & $-0.7289(23)$ & $-0.7120(23)$ & $-0.7281(23)$ & \\
\hline Chongqing & $-0.5080(18)$ & $-0.4674(17)$ & $-0.4880(18)$ & $-0.4150(17)$ & \\
\hline Sichuan & $0.4332(8)$ & $0.4107(8)$ & $0.3137(10)$ & $0.2637(9)$ & \\
\hline Guizhou & $-0.9822(27)$ & $-1.0089(27)$ & $-1.0643(27)$ & $-1.0037(29)$ & \\
\hline Yunnan & $-0.6080(19)$ & $-0.5968(19)$ & $-0.5990(19)$ & $-0.6796(21)$ & \\
\hline Shanxi & $-0.4179(17)$ & $-0.4696(18)$ & $-0.3005(15)$ & $-0.2705(15)$ & \\
\hline Gansu & $-0.8497(25)$ & $-0.9135(25)$ & $-0.9303(25)$ & $-0.8273(25)$ & - \\
\hline Qinghai & $-1.2114(28)$ & $-1.1827(28)$ & $-1.1576(28)$ & $-0.8402(27)$ & \\
\hline Ningxia & $-1.2437(29)$ & $-1.2190(29)$ & $-1.2372(29)$ & $-0.8237(26)$ & \\
\hline Xinjiang & $-0.9162(26)$ & $-0.9285(26)$ & $-0.9558(26)$ & $-0.7671(28)$ & \\
\hline
\end{tabular}

\footnotetext{
*Instructor, School of Management and Economics, Tianjin University, Tianjin, China (E-mail: bluesea_boy_1979@163.com)

**Professor, School of Management and Economics, Tianjin University, Tianjin, China (E-mail: wxq@ $@$ tju.edu.cn)

***Associate Professor, School of Engineering, Zhejiang University City College, Zhejiang, China (Corresponding Author, E-mail: chencl@zucc.edu.cn) ****Director, China Institute of Water Resources and Hydropower Research, Beijing, China (E-mail: mazj88@126.com)
} 
Should be Corrected as:

\begin{tabular}{|c|c|c|c|c|c|}
\hline & 2005 & 2006 & 2007 & 2008 & Trend \\
\hline Beijing & $1.3417(5)$ & $1.1548(5)$ & $1.1451(5)$ & $0.7964(5)$ & - \\
\hline Tianjin & $-0.4023(16)$ & $-0.4226(15)$ & $-0.3919(16)$ & $-0.3884(16)$ & - \\
\hline Hebei & $0.1780(10)$ & $0.1241(11)$ & $0.1031(11)$ & $0.0390(11)$ & $\downarrow$ \\
\hline Shanxi & $-0.3914(15)$ & $-0.4604(16)$ & $-0.4502(17)$ & $-0.4236(18)$ & $\downarrow$ \\
\hline Inner Mongolia & $-0.7310(23)$ & $-0.6884(22)$ & $-0.6963(22)$ & $-0.7443(24)$ & $\downarrow$ \\
\hline Liaoning & $0.5201(7)$ & $0.4597(7)$ & $0.4644(7)$ & $0.3884(8)$ & $\downarrow$ \\
\hline Jilin & $-0.6969(21)$ & $-0.7383(24)$ & $-0.7317(24)$ & $-0.7103(22)$ & $\downarrow$ \\
\hline Heilongjiang & $-0.7011(22)$ & $-0.6579(20)$ & $-0.6941(21)$ & $-0.6308(19)$ & $\uparrow$ \\
\hline Shanghai & $0.7601(6)$ & $0.7473(6)$ & $0.5989(6)$ & $0.5979(6)$ & - \\
\hline Jiangsu & $2.6186(1)$ & $2.7535(1)$ & $2.8260(1)$ & $3.0233(1)$ & - \\
\hline Zhejiang & $1.7875(2)$ & $1.8626(2)$ & $1.8101(2)$ & $1.7941(2)$ & - \\
\hline Anhui & $-0.1650(14)$ & $-0.1112(14)$ & $-0.0933(14)$ & $-0.0412(14)$ & - \\
\hline Fujian & $-0.1318(13)$ & $-0.0169(12)$ & $0.0287(12)$ & $0.0314(12)$ & $\uparrow$ \\
\hline Jiangxi & $-0.6568(20)$ & $-0.6638(21)$ & $-0.6877(20)$ & $-0.6736(20)$ & - \\
\hline Shandong & $1.6190(4)$ & $1.5790(4)$ & $1.4998(4)$ & $1.5355(4)$ & - \\
\hline Henan & $0.1618(11)$ & $0.2956(9)$ & $0.4355(8)$ & $0.5434(7)$ & $\uparrow$ \\
\hline Hubei & $0.2331(9)$ & $0.2628(10)$ & $0.3351(9)$ & $0.2533(10)$ & $\downarrow$ \\
\hline Hunan & $-0.0662(12)$ & $-0.0460(13)$ & $-0.0644(13)$ & $-0.0374(13)$ & $\downarrow$ \\
\hline Guangdong & $1.7787(3)$ & $1.6706(3)$ & $1.6938(3)$ & $1.6539(3)$ & - \\
\hline Guangxi & $-0.7521(24)$ & $-0.7289(23)$ & $-0.7120(23)$ & $-0.7281(23)$ & $\uparrow$ \\
\hline Chongqing & $-0.5080(18)$ & $-0.4674(17)$ & $-0.4880(18)$ & $-0.4150(17)$ & $\uparrow$ \\
\hline Sichuan & $0.4332(8)$ & $0.4107(8)$ & $0.3137(10)$ & $0.2637(9)$ & $\downarrow$ \\
\hline Guizhou & $-0.9822(27)$ & $-1.0089(27)$ & $-1.0643(27)$ & $-1.0037(29)$ & $\downarrow$ \\
\hline Yunnan & $-0.6080(19)$ & $-0.5968(19)$ & $-0.5990(19)$ & $-0.6796(21)$ & $\downarrow$ \\
\hline Shanxi & $-0.4179(17)$ & $-0.4696(18)$ & $-0.3005(15)$ & $-0.2705(15)$ & $\uparrow$ \\
\hline Gansu & $-0.8497(25)$ & $-0.9135(25)$ & $-0.9303(25)$ & $-0.8273(25)$ & - \\
\hline Qinghai & $-1.2114(28)$ & $-1.1827(28)$ & $-1.1576(28)$ & $-0.8402(27)$ & $\uparrow$ \\
\hline Ningxia & $-1.2437(29)$ & $-1.2190(29)$ & $-1.2372(29)$ & $-0.8237(26)$ & $\uparrow$ \\
\hline Xinjiang & $-0.9162(26)$ & $-0.9285(26)$ & $-0.9558(26)$ & $-0.7671(28)$ & $\downarrow$ \\
\hline
\end{tabular}

2. Table 6, Trends of Influence Factors of the Competitiveness of China's Construction Industry:

\begin{tabular}{|c|c|c|c|c|c|c|c|}
\hline & $\begin{array}{c}\text { Economic } \\
\text { benefits }\end{array}$ & $\begin{array}{l}\text { Factor } \\
\text { inputs }\end{array}$ & $\begin{array}{c}\text { Demand } \\
\text { conditions }\end{array}$ & $\begin{array}{l}\text { Auxiliary } \\
\text { industries }\end{array}$ & $\begin{array}{l}\text { Industrial } \\
\text { structure }\end{array}$ & $\begin{array}{l}\text { Production } \\
\text { efficiency }\end{array}$ & $\begin{array}{l}\text { Innovation } \\
\text { factors }\end{array}$ \\
\hline Beijing & & & & - & - & & \\
\hline Tianjin & & & & & - & & \\
\hline Hebei & & & & - & & & \\
\hline Shanxi & & - & & & & & \\
\hline Inner Mongolia & - & - & & - & & & \\
\hline Liaoning & & - & - & - & & & - \\
\hline Jilin & & - & & & & & \\
\hline \multicolumn{8}{|l|}{ Heilongjiang } \\
\hline Shanghai & - & & & & & & \\
\hline Jiangsu & & - & & & & & \\
\hline Zhejiang & & - & - & 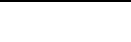 & & & - \\
\hline Anhui & - & - & & . & & & \\
\hline Fujian & & & & - & & & \\
\hline Jiangxi & & & & & & & - \\
\hline Shandong & - & - & - & - & & & \\
\hline Henan & & & - & & & & \\
\hline Hubei & & & & - & & & \\
\hline Hunan & - & & & - & & & \\
\hline Guangdong & - & - & & & & & \\
\hline
\end{tabular}




\begin{tabular}{|c|c|c|c|c|c|c|c|}
\hline Guangxi & - & & & - & & & - \\
\hline Chongqing & & - & & & - & - & \\
\hline Sichuan & & & - & & & - & \\
\hline Guizhou & - & - & - & - & & & \\
\hline Yunnan & & & & - & - & & \\
\hline \multicolumn{8}{|l|}{ Shanxi } \\
\hline Gansu & - & & & - & & - & \\
\hline Ningxia & - & - & & & - & & \\
\hline Xinjiang & - & - & & - & & & - \\
\hline
\end{tabular}

Should be Corrected as:

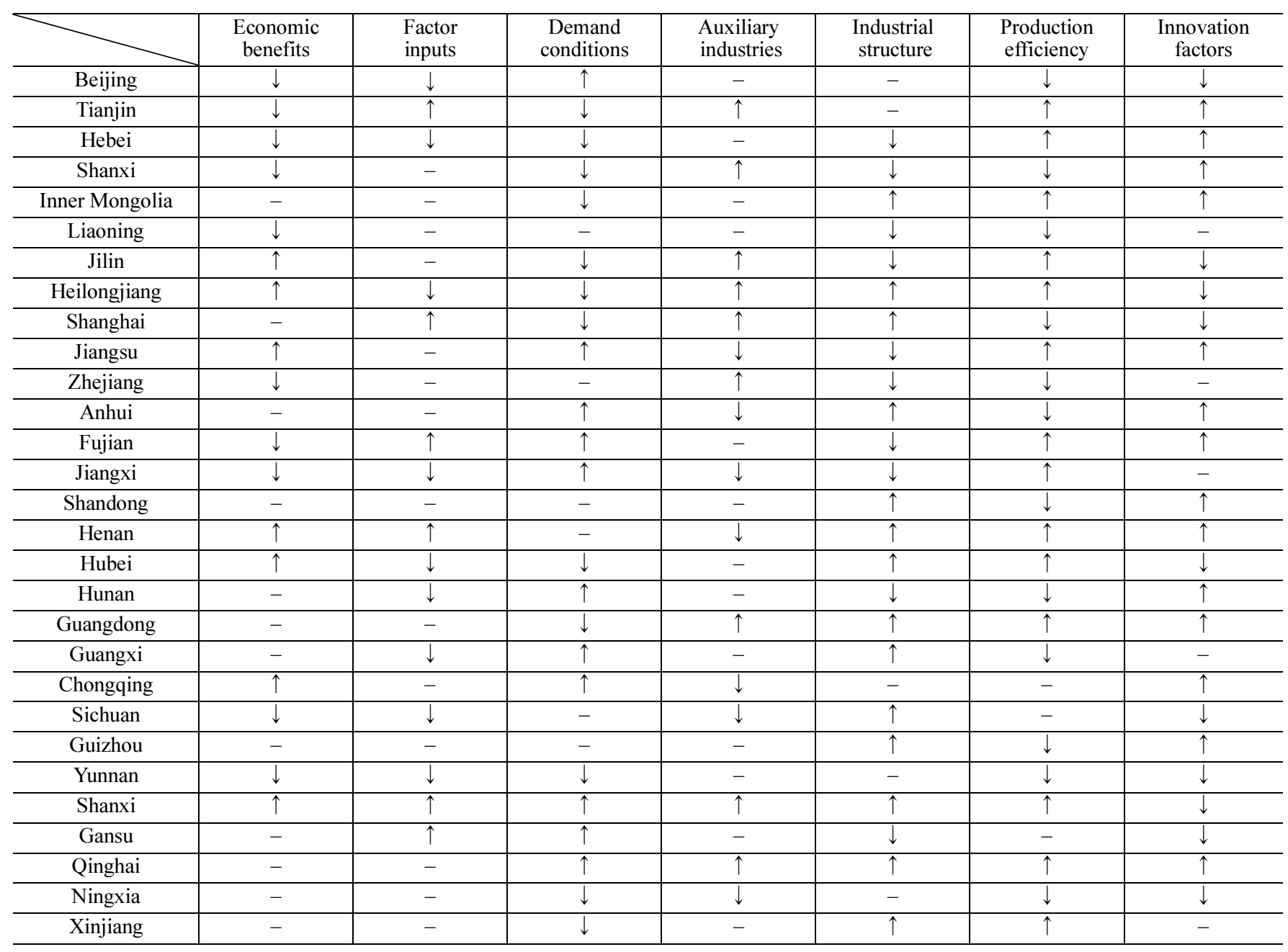

